Proceedings of the 2009 Winter Simulation Conference

M. D. Rossetti, R. R. Hill, B. Johansson, A. Dunkin, and R. G. Ingalls, eds.

\title{
OPTIMAL GENERATION EXPANSION PLANNING VIA THE CROSS-ENTROPY METHOD
}

\author{
Rishabh P. Kothari \\ Management Science and Engineering \\ Stanford University \\ Stanford, CA 94305, U.S.A.
}

\author{
Dirk P. Kroese \\ Department of Mathematics \\ University of Queensland \\ Brisbane 4072, Australia
}

\begin{abstract}
The Generation Expansion Planning (GEP) problem is a highly constrained, large-scale, mixed integer nonlinear programming problem. The objective of the GEP problem is to evaluate the least cost investment plan for addition of power generating units over a planning period subject to demand, availability, and security constraints. In this paper, a GEP model is presented and the Cross-Entropy (CE) optimization method is developed to solve the problem. The CE method is an effective algorithm for solving large combinatorial optimization problems. The main advantage of the CE method over other metaheuristic techniques is that it does not require decomposition of the problem into a master problem and operation subproblems, greatly reducing the computational complexity. This method also provides a fast and reliable convergence to the optimal solution.
\end{abstract}

\section{INTRODUCTION}

The electric power industries worldwide have been witnessing tremendous growth, especially in developing nations. Optimum investment policies for addition of new generation utilities in order to satisfy increased demands have to be determined. Decisions like when to commission what kind of utilities are essential. In order to facilitate planners, several deterministic (Turvey and Anderson 1977, Anderson 1972) as well as stochastic models (Park, Lee, and Youn 1985, Mankki 1991, Mo, Hegge, and Wangensteen 1991, Gorenstin et al. 1993) have been proposed to simulate generation expansion planning. Stochastic models consider uncertainties in future demand, fuel costs etc. Deterministic models are used to evaluate plans for a number of predetermined scenarios. Game theoretic models (Chuang, Wu, and Varaiya 2001) have also been developed to simulate competition within industry.

The objective of the GEP problem is to determine the minimum cost plan for setting up power generation utilities in order to satisfy future electricity demand. The plan includes decisions such as type of plant, capacity, time of introduction, and the utilization of each plant in the following years. For example, a planner may choose from a $2 \mathrm{MW}$ windmill which can only supply peaking power, or a 2000MW nuclear plant for base power. Each plan has to be devised under several constraints like reliabilty, demand and required fuel mix. These constraints ensure that the proposed plans ensure a stable supply to the required reliability levels. For example, a plan must have an appropriate mix of coal, hydro, gas, wind and other technologies, as supply of any one kind of fuel is unreliable. Apart from the cost objective, these models have been extended to a multicriteria optimization as well. Minimization of environmental costs, or a combination of the environmental and economic costs may be carried out.

GEP models are large scale and higly constrained, and may have discrete as well as continous decision variables. A number of methodologies including linear programming (Turvey and Anderson 1977, Climaco et al. 1995), nonlinear programming (Turvey and Anderson 1977), decomposition methods (Dantzig et al. 1989), dynamic programming (Ryuya Tanabe 1992), expert systems (David and Rongda 1991), fuzzy logic (Satoh and Serizawa 1989), immune algorithms (Chen, Zhan, and Tsay 2006), simulated annealing (Yildirim, Erkan, and Ozturk 2006), particle swarm optimization (Kannan et al. 2004) and genetic algorithms (Park, Park, and Won 1998, Sirikum and Techanitisawad 2006) have been used to solve the GEP problem. A comparison of metaheuristic techniques is presented in (Kannan, Slochanal, and Padhy 2005). Commercial packages such as WASP (Jenkins and Joy 1974) and EGEAS (Caramanis, Schweppe, and Tabors 1982) have also been developed using dynamic programming with heuristic tunneling techniques. 


\section{Kothari and Kroese}

In this paper we present a new method to solve the GEP problem. The Cross-Entropy method (Rubinstein and Kroese 2004) was originally devised as an algorithm for rare event simulation (Rubinstein 1997). Later, it was also proven to be a simple and effective algorithm suited to solve both stochastic and deterministic combinatorial optimization problems (Rubinstein 2001). It has also been extended to solving continuous multi-extremal optimization problems (Kroese, Porotsky, and Rubinstein 2006). The CE method is witnessing an increasing number of applications including queing networks (de Boer 2000), reliability systems (Kroese, Hui, and Nariai 2007), vehicle routing (Chepuri and Homem-de Mello 2005), optimal control (Sani and Kroese 2008) etc. Several applications of the CE method have reported better and faster solutions than other randomized algorithms. Recently, effective parallel computation implementations (Evans, Keith, and Kroese 2007) have been introduced, improving the performance manifold. It has also been presented to the power systems community as an effective tool for solving the unit commitment problem as well (Ernst et al. 2007).

Traditionally, GEP problems have been solved using dynamic programming by decomposing it into a master problem and a number of subproblems. The master problem determines the optimal investment for setting up new utilities, while the subproblems determine the least operating cost for the utilities, given the demand, availability, and reliability constraints. In this paper we propose a simple and efficient implementation of the Cross-Entropy method for solving the GEP problem without resorting to decomposition heuristics. It will also be evident that the $\mathrm{CE}$ method does not require much tweaking of its parameters to suit different problems. Hence, it is a problem independent procedure, unlike other optimization metaheuristics.

The rest of this paper is organized as follows. Section 2 presents the GEP model used. Section 3 provides a brief overview of the CE methodology and also shows how it is applied to the GEP problem. Finally, we apply the CE method to a synthetic GEP problem and provide the results.

\section{PROBLEM FORMULATION}

The problem discussed in this paper is based on the deterministic GEP model of (Turvey and Anderson 1977), as presented in (Sirikum and Techanitisawad 2006). This particular model has been used as it may incorporate more advanced constraints like location, pollution and pollutant concentration; and may also include Demand Side Management(DSM) programs. However, since our focus is on presenting the effectiveness of the CE method as a viable alternative technique, we have neglected the less important constraints.

Apart from the initial investment costs, the parameters include a forecasted demand curve, a load duration curve, the variable fuel and maintenance costs, the discount rate, the allowable loss of load probability, the desired reserve margin, as well as plant characteristics such as availability and capacity factor. Relevant nomenclature is presented in Appendix A.

A simplified problem is formulated as follows.

\subsection{GEP Model}

Define a vector $Y_{n t}$ as:

$$
Y_{n t}=\left\{\begin{array}{l}
1, \text { if unit } n \text { is set up at or before year } t, \\
0, \text { otherwise. }
\end{array}\right.
$$

Objective function: Minimise the total discounted $\operatorname{cost} Z$,

$$
Z=\sum_{t=1}^{T} \sum_{n=1}^{N} w_{t}\left(i_{n t}-s_{n t}\right) \cdot Y_{n t}+\sum_{p=1}^{P} \sum_{t=1}^{T} \sum_{n=1}^{N}\left(f_{n t p}+v_{n t p}\right) \cdot G_{n t p} \cdot d_{p},
$$

subject to the following constraints:

1. Power demand constraint:

$$
(1-l) \cdot\left(\sum_{n=1}^{N} G_{n t p}\right) \geq q_{t p}, \quad \forall t, p .
$$

2. Capacity constraint:

$$
G_{n t p} \leq a_{n t} \cdot Y_{n t} \cdot p_{n t}, \quad \forall t, n, p .
$$




\section{Kothari and Kroese}

3. Thermal energy availability constraint:

$$
\sum_{p=1}^{P}\left(G_{n t p} \cdot d_{p}\right) \leq 8760 \cdot c_{n} \cdot Y_{n t} \cdot p_{n t}, \quad \forall t, n
$$

4. Reliability constraints:

(a) Reserve margin constraint:

$$
(1-l) \cdot\left(\sum_{n=1}^{N} a_{n t} \cdot X_{n t} \cdot p_{n t}\right) \geq(1+R) \cdot q_{t p}, \quad \forall t, p .
$$

(b) LOLP constraint:

$$
\varepsilon_{t} \leq \varepsilon_{t}^{*}, \quad \forall t
$$

5. Non-negativity constraints

$$
\begin{gathered}
G_{n t p} \geq 0, \quad \forall n, t, p . \\
X_{n t} \quad \text { Binary. }
\end{gathered}
$$

The objective function (1) is the sum of the discounted (i.e. present worth) of capital costs, fuel costs and the maintenance costs. The present worth of any distributed investment is calculated as

$$
\mathrm{PW}=\sum_{t=0}^{N} \frac{C_{t}}{(1+r)^{t}}
$$

where $C_{t}$ and $r$ represent the investment and the discount rate for year $t$, respectively. Hence, the discount factor is defined as $w_{t}=(1+r)^{-t}$.

The power demand constraint (2) ensures that the net available power is greater than the demand for each season. The capacity constraint (3) restricts the power production of each plant below its capacity. Fuel availability can be included in the thermal energy constraint (4). Reserve margin required above the peak demand is included in (5), while the LOLP limit can be applied using (6). The LOLP is calculated using the cumulant method as described in (Stremel et al. 1980) and also applied in (Sirikum and Techanitisawad 2006). It is the source of the nonlinearity of the problem.

It must be realised that the disadvantage of this model is that each additional candidate plant increases the dimension of the solution space. On the other hand, this also affords the planner the flexibility to parameterise each plant independently, rather than create sets of plants based on common plant characteristics like fuel used. The limit on any particular type of plant may be applied by limiting the number of candidate plants, or by explicitly defining a constraint in the program.

\section{THE CROSS ENTROPY METHOD}

\subsection{Overview of the $\mathrm{CE}$ method}

The Cross-Entropy(CE) method is a novel Monte Carlo method that has proven successful for solving combinatorial optimisation problems. It is a simple and versatile algorithm suited to a large number of problems. The CE tutorial (de Boer et al. 2004) is a gentle introduction to this optimisation technique. Essentially, the CE method is an iterative Monte Carlo procedure in which each iteration can be described as follows:

1. Generate a random data sample according to a specified mechanism.

2. Update the parameters of the random mechanism based on the data to produce a "better" sample in the next iteration. This step involves a cross-entropy minimisation procedure. 


\section{Kothari and Kroese}

The CE method is rooted in well known statistical principles. It uses the Importance Sampling(IS) technique by minimising the Kullback-Leibler, or cross-entropy, distance between the optimal zero-variance measure pdf and the chosen importance sampling distribution.

A detailed derivation is presented in (Rubinstein and Kroese 2004). Suppose we wish to maximise the function $Z(\mathbf{x})$, where $\mathbf{x}$ is a vector or state in set $\mathscr{X}$. Let us denote the maximum by $\gamma^{*}$. Thus,

$$
\gamma^{*}=\max _{\mathbf{x} \in \mathscr{X}} Z(\mathbf{x})=Z\left(\mathbf{x}^{*}\right)
$$

Now, instead of searching for $\mathbf{x}^{*}$ directly in the set $\mathscr{X}$, we define a family of pdf's $f(\cdot ; \mathbf{v}), \mathbf{v} \in \mathscr{V}$ on $\mathscr{X}$ and solve a related estimation problem, called the associated stochastic problem, namely, the estimation of the probability:

$$
\ell(\gamma)=\mathbb{P}_{\mathbf{u}}(Z(\mathbf{X}) \geq \gamma)=\mathbb{E}_{\mathbf{u}} I_{\{Z(\mathbf{X}) \geq \gamma\}}
$$

where $\mathbf{X}$ is a random vector with pdf $f(\cdot, \mathbf{u})$, for some $\mathbf{u} \in \mathscr{V}$, and $\gamma$ is left unspecified. For $\gamma \approx \gamma^{*}, \ell$ will be very small and under the optimal IS measure, $\mathbf{X} \approx \mathbf{x}^{*}$. The CE method works by adaptively changing the probability density parameter $\mathbf{v}$ towards the optimal density $\mathbf{v}^{*}$. This results in a sequence of tuples $\left(\hat{\gamma}_{t}, \hat{\mathbf{v}}_{t}\right)$ which converge quickly to a "degenerate" optimal tuple $\left(\hat{\gamma}^{*}, \hat{\mathbf{v}}^{*}\right)$.

The CE method is initialised by choosing an initial $\hat{\mathbf{v}}_{0} . N$ represents the number of samples generated in each iteration. It is assumed to be a fixed number. The parameter $\rho$ represents the fraction of "best" performing samples, and is used to calculate $\gamma$. Usually, $\rho$ is of the order of $10^{-2}$. The iterative procedure is as follows:

1. Adaptive updating of $\gamma_{t}$. For a fixed $\mathbf{v}_{t-1}$, let $\gamma_{t}$ be the $(1-\rho)$-quantile of $Z(\mathbf{X})$ under $\mathbf{v}_{t-1}$. That is, $\gamma_{t}$ satisfies

$$
\begin{gathered}
\mathbb{P}_{\mathbf{v}_{t-1}}\left(Z(\mathbf{X}) \geq \gamma_{t}\right) \geq \rho, \\
\mathbb{P}_{\mathbf{v}_{t-1}}\left(Z(\mathbf{X}) \leq \gamma_{t}\right) \geq 1-\rho .
\end{gathered}
$$

A simple estimator $\hat{\gamma}_{t}$ of $\gamma_{t}$ can be obtained by taking a random sample $\mathbf{X}_{1}, \mathbf{X}_{2}, \ldots, \mathbf{X}_{N}$ from $f\left(\cdot ; \mathbf{v}_{t-1}\right)$ and evaluating the sample $(1-\rho)$-quantile of performances as:

$$
\hat{\gamma}_{t}=Z_{(\lceil(1-\rho) N\rceil)},
$$

where $Z_{(1)} \leq Z_{(2)} \leq \ldots \leq Z_{(N)}$

2. Adaptive updating of $\mathbf{v}_{t}$. For fixed $\gamma_{t}$ and $\mathbf{v}_{t-1}$, derive $\mathbf{v}_{t}$ from the solution of the cross-entropy program:

$$
\max _{\mathbf{v}} D(\mathbf{v})=\max _{\mathbf{v}} \mathbb{E}_{\mathbf{v}_{t-1}} \mathbb{I}_{\left\{Z(\mathbf{X}) \geq \gamma_{t}\right\}} \ln f(\mathbf{X} ; \mathbf{v})
$$

The stochastic counterpart of equation (8) is as follows: for fixed $\hat{\gamma}_{t}$ and $\hat{\mathbf{v}}_{t-1}$, derive $\hat{\mathbf{v}}_{t}$ from the following program:

$$
\max _{\mathbf{v}} \hat{D}(\mathbf{v})=\max _{\mathbf{v}} \frac{1}{N} \sum_{i=1}^{N} \mathbb{I}_{\left\{Z\left(\mathbf{X}_{i}\right) \geq \hat{\gamma}_{t}\right\}} \ln f\left(\mathbf{X}_{i} ; \mathbf{v}\right)
$$

Remark In order to slow the convergence to avoid a sub-optimal solution, we use the following smoothed updating instead of the above solution:

$$
\hat{\mathbf{v}}_{t}=\alpha \tilde{\mathbf{v}}_{t}+(1-\alpha) \hat{\mathbf{v}}_{t-1}
$$

where $\tilde{\mathbf{v}}_{t}$ is the parameter obtained from (9). $\alpha$ is called the smoothing parameter and usually $0.7 \leq \alpha \leq 1$. A detailed explanation of smoothing procedures is available in (Kroese, Porotsky, and Rubinstein 2006).

Typically, the sampling pdf is chosen such that it belongs to a Natural Exponential Family (e.g., Gaussian or Bernoulli). This enables an analytical solution of (9). Refer to (Rubinstein and Kroese 2004) for further explanation. Algorithm 1 summarises how the samples are generated and updated to result in a optimal solution. 


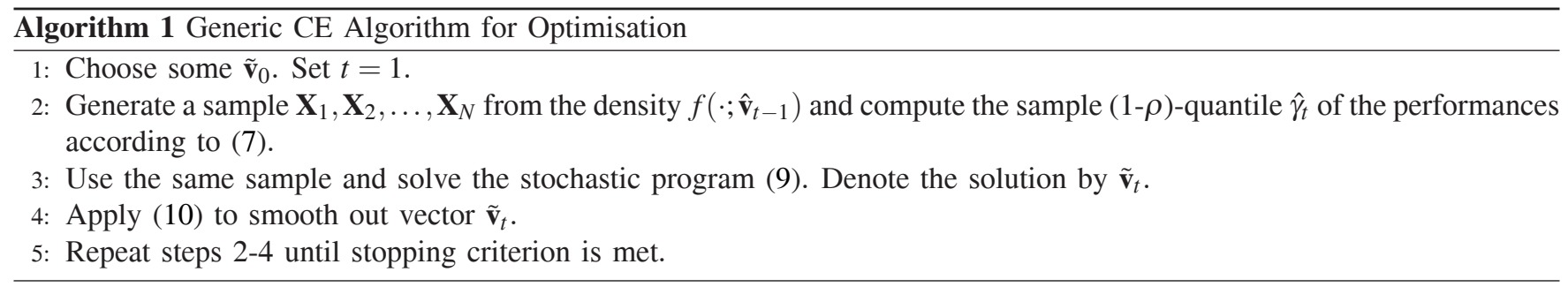

\subsection{CE Method for GEP}

In the presented model of the GEP, both discrete and continuous decision variables have to be worked with. Let $\mathbf{X}$ be the vector of binary decision variables $X_{n t}$. Recall that each element $X_{n t}$ represents whether or not candidate plant $n$ has been set up at year $t$. At each stage of the $\mathrm{CE}$ algorithm the sampling pdf for $\mathbf{X}$ is multi-variate Bernoulli with success probability vector $\hat{\mathbf{p}}$. We write $\mathbf{X} \sim \operatorname{Ber}(\hat{\mathbf{p}})$. In particular, the components of $\mathbf{X}$ are independent, and

$$
\sum_{j=0}^{T} \mathbb{P}\left(X_{n j}=1\right)=\sum_{j=0}^{T} \hat{p}_{n j}=1 .
$$

We have assumed $\hat{p}_{n 0}$ to be the possibility of not selecting plant $n$ at all.

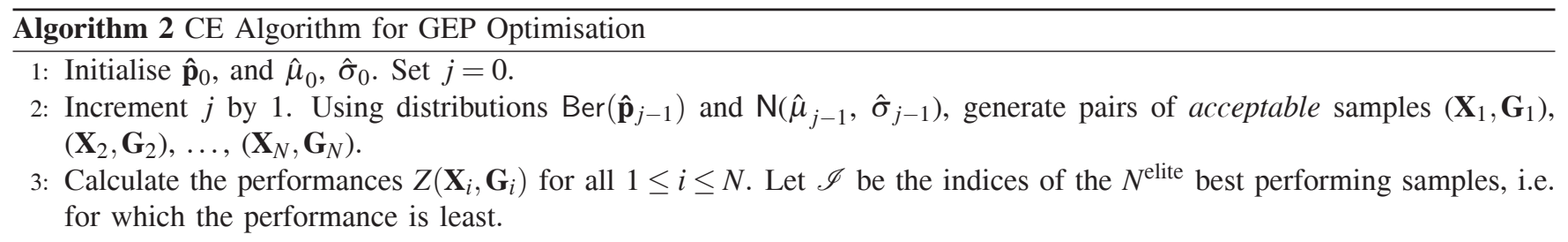

4: Update the parameters:

$$
\begin{gathered}
\tilde{p}_{j n t}=\frac{\sum_{k=1}^{N} \mathbb{I}_{\{k \in \mathscr{I}\}} \mathbb{I}_{\left\{X_{k n t}=1\right\}}}{N^{\text {elite }}}, \quad \forall n, t \\
\tilde{\mu}_{\text {jntp }}=\frac{\sum_{i \in \mathscr{I} G_{\text {int }}}}{N^{\text {elite }}}, \quad \forall n, t, p .
\end{gathered}
$$

and

$$
\tilde{\sigma}_{j n t p}=\frac{\sum_{i \in \mathscr{I}}\left(G_{i n t p}-\tilde{\mu}_{j n t p}\right)^{2}}{N^{\text {elite }}} \cdot \quad \forall n, t, p .
$$

5: Smooth:

$$
\begin{aligned}
& \hat{\mathbf{p}}_{j}=\alpha \tilde{\mathbf{p}}_{j}+(1-\alpha) \hat{\mathbf{p}}_{j-1}, \\
& \hat{\mu}_{j}=\alpha \tilde{\mu}_{j}+(1-\alpha) \hat{\mu}_{j-1}, \\
& \hat{\sigma}_{j}=\alpha \tilde{\sigma}_{j}+(1-\alpha) \hat{\sigma}_{j-1} .
\end{aligned}
$$

6: Repeat from step 2 until stopping criterion is met.

The second decision vector $\mathbf{G}$ represents the utilisation of each plant. Each element $G_{n t p}$ is sampled from a Gaussian distribution $\mathrm{N}\left(\hat{\mu}_{n t p}, \hat{\sigma}_{n t p}\right)$. Algorithm 2 shows the CE algorithm for the GEP problem. In the course of the optimisation, each $\mathrm{N}\left(\hat{\mu}_{n t p}, \hat{\sigma}_{n t p}\right)$ should converge to a degenerate distribution with the final $\hat{\mu}_{n t p}$ being the resultant utilisation for each 


\section{Kothari and Kroese}

season. A possible stopping criterion is when $\max \sigma_{n t p} \leq \delta$, where $\delta \approx 0.1$, or when $\hat{\gamma}_{t}$ does not change for a few iterations. Figure 4 shows the convergence of $\max \sigma_{n t p}$ for a sample run of the GEP program.

\section{NUMERICAL RESULTS}

The computer program for the $\mathrm{CE}$ method implementation is developed in $\mathrm{C}++$. The tests are carried out on a $3 \mathrm{GHz}$ Linux machine with 1 GB RAM.

\subsection{Test System Descriptions}

The proposed CE method was applied to a synthetic test problem. A 10 year planning period with stages at 1-year intervals is considered. Table 2 shows that data for the existing plants. Table 1 lists the candidate plants.

\subsection{GEP and CE Parameters}

The peak power demand is considered to be $1600 \mathrm{MW}$ at year zero with a $10 \%$ annual rise. The load duration curve is simplified as shown in Figure 1. It is discretized into 3 parts. LOLP limit is taken to be 0.01 , the discount rate is $8.5 \%$. System losses are 5\% of the produced power, while the reserve margin is $15 \%$ of the peak load. The CE parameters required no tweaking and were kept constant for all the runs. The number of samples was set at 2,000 and $\rho$ was set to 0.05 . The stopping criterion was chosen as $\max \sigma_{n t p}<0.01$.

Table 1: Data of candidate plants

\begin{tabular}{|l|c|c|c|c|c|}
\hline Type & Capacity & FOR & $\begin{array}{c}\text { Cap. } \\
\text { cost } \\
(\mathbf{M W})\end{array}$ & $\begin{array}{c}\text { Fuel } \\
\text { cost } \\
(\$ / \mathrm{MWh})\end{array}$ & $\begin{array}{c}\text { Maint. } \\
\text { cost } \\
(\$ / \mathrm{kW})\end{array}$ \\
\hline P1:Coal & 1000 & 6 & 735 & 4.21 & 30 \\
P2:Oil & 300 & 8 & 341 & 11.30 & 30 \\
P3:Oil & 300 & 8 & 341 & 11.30 & 30 \\
P4:Oil & 700 & 6 & 390 & 9.24 & 30 \\
P5:Oil & 700 & 6 & 390 & 9.24 & 30 \\
P6:Lignite & 300 & 8 & 400 & 9.88 & 30 \\
P7:Lignite & 300 & 8 & 400 & 9.88 & 30 \\
P8:Lignite & 300 & 8 & 400 & 9.88 & 30 \\
P9:Lignite & 300 & 8 & 400 & 9.88 & 30 \\
P10:Lignite & 300 & 8 & 400 & 9.88 & 30 \\
P11:Gas & 300 & 6 & 152 & 12.16 & 30 \\
P12:Gas & 300 & 6 & 152 & 12.16 & 30 \\
\hline
\end{tabular}

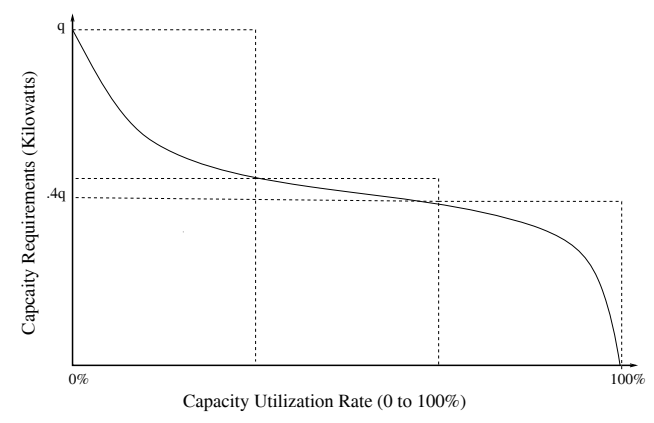

Figure 1: Load duration curve, $P=3$ 


\section{Kothari and Kroese}

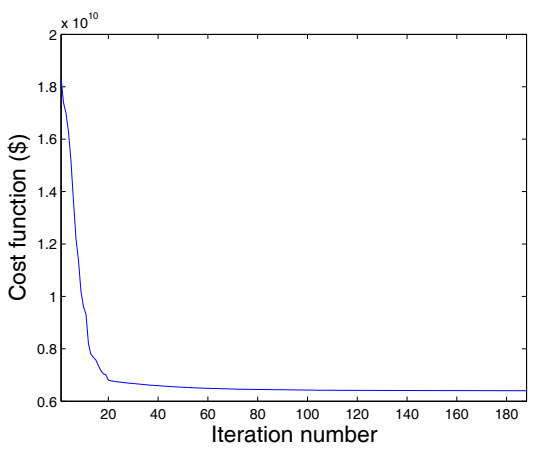

Figure 2: Convergence of the cost function, $Z$

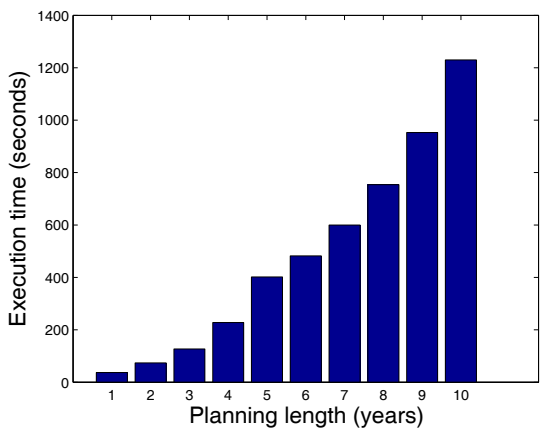

Figure 3: Execution time for different planning periods

Table 2: Data of existing plants

\begin{tabular}{|l|c|c|c|c|c|}
\hline Type & Capacity & FOR & $\begin{array}{c}\text { Cap. } \\
\text { cost } \\
(\$ / \mathrm{kW})\end{array}$ & $\begin{array}{c}\text { Fuel } \\
\text { cost } \\
(\$ / \mathrm{MWh})\end{array}$ & $\begin{array}{c}\text { Maint. } \\
\text { cost } \\
(\$ / \mathrm{kW})\end{array}$ \\
\hline E1:Coal & 1000 & 6 & 7350 & 4.21 & 30 \\
E2:Oil & 300 & 8 & 3410 & 11.30 & 30 \\
E3:Oil & 700 & 6 & 3900 & 9.24 & 30 \\
E4:Lignite & 300 & 8 & 4000 & 9.88 & 30 \\
E5:Gas & 300 & 6 & 1520 & 12.16 & 30 \\
\hline
\end{tabular}

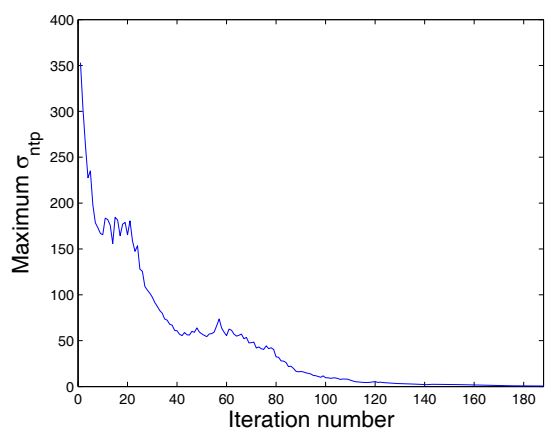

Figure 4: Convergence of $\sigma$ for $N=2000, \rho=0.05$

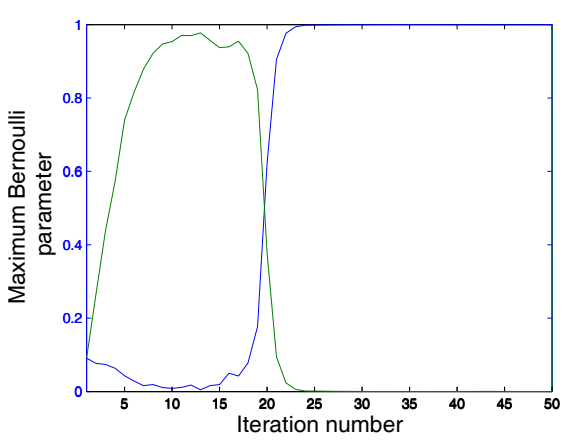

Figure 5: Convergence of Bernoulli probabilities for $N=2000$, $\rho=0.05$

\subsection{Numerical Results}

The results for a sample 10 year run of the CE implementation are presented in Table 3. As expected, the simulation prefers to set up the larger plants, with lower running costs, earlier than the smaller peaking plants. Figure 2 shows the convergence of the cost function, while Figure 4 displays the convergence of the maximum standard deviation, max $\hat{\sigma}_{n t p}$, for each iteration. Figure 5 displays the convergence of $\hat{p}_{n t}$. It represents the values farthest away from their respective final values, at each run. It can be seen that the $\mathrm{CE}$ algorithm quickly solves the unit selection master problem, and then converges to optimal values of the subproblem. It was also noticed that the CE method provided the optimal solution 7 out of 10 times, with runtimes and deviations as shown in Table 4. Figure 3 displays the execution time for different number of stages.

The CE program also compares favourably with other techniques applied to GEP optimisation. A higher repeatability is achieved, while execution times are comparable to prior works (Sirikum, Techanitisawad, and Kachitvichyanukul 2007). 
Table 3: Results of simulation

\begin{tabular}{|c|c|c|c|c|c|c|c|c|c|c|c|c|}
\hline Plant & Capacity & \multicolumn{8}{|c|}{ Utilisation at peak demand for each year (MW) } \\
\cline { 3 - 12 } & (MW) & 1 & 2 & 3 & 4 & 5 & 6 & 7 & 8 & 9 & 10 \\
\hline E1 & 1000 & 836.495 & 781.644 & 775.421 & 798.636 & 797.505 & 787.941 & 866.351 & 843.138 & 863.197 & 892.644 \\
E2 & 300 & 196.29 & 104.485 & 172.302 & 185.022 & 148.97 & 128.368 & 131.08 & 186.017 & 184.115 & 214.034 \\
E3 & 700 & 559.018 & 426.252 & 485.46 & 514.363 & 490.868 & 457.213 & 502.492 & 514.488 & 516.679 & 578.014 \\
E4 & 300 & 213.396 & 174.912 & 146.728 & 152.041 & 176.544 & 160.679 & 174.233 & 178.462 & 197.407 & 213.13 \\
E5 & 300 & 150.787 & 118.004 & 146.98 & 144.714 & 156.449 & 175.512 & 170.805 & 148.31 & 168.541 & 228.913 \\
\hline P1 & 300 & 0 & 0 & 0 & 0 & 0 & 0 & 0 & 0 & 0 & 0 \\
P2 & 300 & 0 & 0 & 0 & 0 & 0 & 0 & 0 & 0 & 0 & 0 \\
P3 & 300 & 0 & 0 & 0 & 0 & 0 & 0 & 0 & 0 & 0 & 0 \\
P4 & 700 & 0 & 0 & 0 & 0 & 0 & 288.462 & 407.952 & 467.154 & 513.135 & 590.679 \\
P5 & 300 & 0 & 0 & 0 & 0 & 0 & 0 & 0 & 0 & 168.803 & 200.952 \\
P6 & 1000 & 0 & 546.433 & 639.955 & 808.693 & 686.508 & 781.737 & 783.258 & 820.076 & 869.49 & 897.562 \\
P7 & 300 & 0 & 0 & 0 & 0 & 0 & 0 & 0 & 0 & 0 & 0 \\
P8 & 300 & 0 & 0 & 0 & 0 & 0 & 0 & 0 & 154.206 & 154.555 & 204.458 \\
P9 & 300 & 0 & 0 & 0 & 0 & 0 & 0 & 0 & 0 & 0 & 0 \\
P10 & 300 & 0 & 0 & 0 & 0 & 0 & 0 & 0 & 0 & 0 & 0 \\
P11 & 700 & 0 & 0 & 0 & 0 & 406.803 & 370.097 & 428.95 & 499.687 & 556.551 & 591.459 \\
P12 & 300 & 0 & 0 & 0 & 0 & 0 & 0 & 0 & 0 & 0 & 0 \\
\hline
\end{tabular}

Table 4: Summary of results for 10 year execution

\begin{tabular}{|c|c|c|c|c|c|c|}
\hline \multirow{2}{*}{ Runs } & \multicolumn{3}{|c|}{ Performance $\left(\$ 10^{9}\right)$} & \multicolumn{3}{c|}{ Runtime (min) } \\
\cline { 2 - 7 } & Best & Worst & Avg. & Best & Worst & Avg. \\
\hline 10 & 6.7897 & 6.8121 & 6.7903 & 21 & 23 & 21 \\
\hline
\end{tabular}

\section{CONCLUSION}

This paper addressed the GEP problem by elucidating a novel CE method toward its solution. The method has proven efficient for large nonlinear discrete and continuous constrained optimisation problems, and has several advantages. There is no need to formulate and solve the operation subproblem. The CE method is capable of optimal multivariate solutions without these heuristics. This greatly reduced the complexity of the program. Another advantage as compared to other numerical optimisation procedures is that the $\mathrm{CE}$ parameters need not be tweaked for each run. The simulation results also show that CE provides for greater repeatability with comparable execution times. This method results in robust solutions for each problem as the algorithm itself is problem independent.

\section{ACKNOWLEDGEMENT}

The authors would like to thank the Australian Research Council (Discovery Grant DP0985177) for supporting this work.

\section{A NOMENCLATURE}

\footnotetext{
Indices

$n \quad$ Generating unit, $(n=1,2, \ldots, N)$.

$p \quad$ Season of load duration curve, $(p=1,2, \ldots, P)$.

$t \quad$ Year of operation of generating units $(t=1,2, \ldots, T)$.

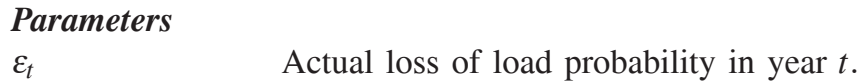

$\varepsilon_{t}^{*} \quad$ Allowable loss of load probability in year $t$.

$a_{n t} \quad$ Availability of generating unit $n$ in year $t(\%)$.
} 


\section{Kothari and Kroese}

$\begin{array}{ll}d_{p} & \text { Duration of season } p \text { (hours). } \\ f_{n t p} & \text { Fuel cost per unit energy output from plant } n \text { in year } t \text { and season } p(\$) . \\ i_{n t} & \text { Investment cost of plant } n \text { in year } t(\$) . \\ l & \text { Transmission and distribution losses }(\%) \\ c_{n} & \text { Annual capacity factor for plant } n(\%) \\ p_{n t} & \text { Power capacity of generating unit } n \text { in year } t \\ q_{t p} & \text { Power demand in season } p \text { in year } t \\ R & \text { Reserve margin }(\%) \\ s_{n t} & \text { Salvage values of generating unit } n \text { in year } t \\ w_{t} & \text { Discount factor }(\%) \\ Z & \text { Total discounted cost function. }\end{array}$

\section{Decision Variables}

$G_{n t p} \quad$ Utilisation of generating unit $n$ in year $t$ and season $p(\mathrm{MW})$.

$X_{n t}$ $\left\{\begin{array}{l}1, \text { if unit } n \text { is set up at year } t, \\ 0, \text { otherwise. }\end{array}\right.$

\section{REFERENCES}

Anderson, D. 1972. Models for determining least-cost investments in electricity supply. The Bell Journal of Economics and Management Science 3 (1): 267-299.

Caramanis, M., F. Schweppe, and R. Tabors. 1982. Electric-generation-expansion analysis system. volume 1: solution techniques, computing methods, and results. final report. United States.

Chen, S.-L., T.-S. Zhan, and M.-T. Tsay. 2006, Jan. Generation expansion planning of the utility with refined immune algorithm. Electric Power Systems Research 76 (4): 251-258.

Chepuri, K., and T. Homem-de Mello. 2005, Feb. Solving the vehicle routing problem with stochastic demands using the cross-entropy method. Annals of Operations Research 134 (1): 153-181.

Chuang, A., F. Wu, and P. Varaiya. 2001, Nov. A game-theoretic model for generation expansion planning: problem formulation and numerical comparisons. Power Systems, IEEE Transactions on 16 (4): 885-891.

Climaco, J., C. H. Antunes, A. G. Martins, and A. T. Almeida. 1995. A multiple objective linear programming model for power generation expansion planning. International Journal of Energy Research 19 (5): 419-432.

Dantzig, G., P. Glynn, M. Avriel, J. Stone, R. Entriken, and M. Nakayama. 1989. Decomposition techniques for multi-area generation and transmission planning under uncertainty: Final report. Pages: 121. United States.

David, A., and Z. Rongda. 1991, Feb. An expert system with fuzzy sets for optimal planning [of power system expansion].

de Boer, P. T. 2000. Analysis and efficient simulation of queueing models of telecommunication systems. Ph. D. thesis, University of Twente.

de Boer, P. T., D. P. Kroese, S. Mannor, and R. Y. Rubinstein. 2004. A tutorial on the cross-entropy method. Annals of Operations Research 134 (1): 19-67.

Ernst, D., M. Glavic, G.-B. Stan, S. Manor, and L. Wehenkel. 2007. The cross-entropy method for power system combinatorial optimization problems. In Proc. IEEE Power Tech Conference, Lausanne, July 2007.

Evans, G. E., J. M. Keith, and D. P. Kroese. 2007. Parallel cross-entropy optimization. In WSC '07: Proceedings of the 39th Winter Simulation Conference, 2196-2202. Piscataway, NJ, USA: IEEE Press.

Gorenstin, B., N. Campodonico, J. Costa, and M. Pereira. 1993, Feb. Power system expansion planning under uncertainty. Power Systems, IEEE Transactions on 8 (1): 129-136.

Jenkins, R., and D. Joy. 1974. Wein automatic system planning package (wasp): an electric utility optimal generation expansion planning computer code. Pages: 108. United States.

Kannan, S., S. Slochanal, and N. Padhy. 2005, Feb.. Application and comparison of metaheuristic techniques to generation expansion planning problem. Power Systems, IEEE Transactions on 20 (1): 466-475.

Kannan, S., S. M. R. Slochanal, P. Subbaraj, and N. P. Padhy. 2004, Aug. Application of particle swarm optimization technique and its variants to generation expansion planning problem. Electric Power Systems Research 70 (3): $203-210$.

Kroese, D. P., K.-P. Hui, and S. Nariai. 2007, June. Network reliability optimization via the cross-entropy method. Reliability, IEEE Transactions on 56 (2): 275-287.

Kroese, D. P., S. Porotsky, and R. Y. Rubinstein. 2006, Sep. The cross-entropy method for continuous multi-extremal optimization. Methodology and Computing in Applied Probability 8 (3): 383-407. 


\section{Kothari and Kroese}

Mankki, P. 1991, Jul. Electric power capacity planning under uncertain conditions. Probabilistic Methods Applied to Electric Power Systems, 1991., Third International Conference on:214-219.

Mo, B., J. Hegge, and I. Wangensteen. 1991, May. Stochastic generation expansion planning by means of stochastic dynamic programming. Power Systems, IEEE Transactions on 6 (2): 662-668.

Park, Y., K. Lee, and L. Youn. 1985, Feb.. New ananlytical approach for long-term generation expansion planning based on maximum principle and gaussian distribution function. IEEE Transactions on Power Apparatus and Systems PAS-104 (2): 390-397.

Park, Y. M., J. B. Park, and J. R. Won. 1998, May. A hybrid genetic algorithm/dynamic programming approach to optimal long-term generation expansion planning. International Journal of Electrical Power \& Energy Systems 20 (4): 295-303.

Rubinstein, R. Y. 1997. Optimization of computer simulation models with rare events. European Journal of Operational Research 99:89-112.

Rubinstein, R. Y. 2001. Combinatorial optimization via cross-entropy. In Encyclopedia of Operations Research and Management Sciences, ed. S. Gass and C. Harris, 102-106: Kluwer.

Rubinstein, R. Y., and D. P. Kroese. 2004. The cross-entropy method. Springer-Verlag.

Ryuya Tanabe, Keiichiro Yasuda, R. Y. 1992. Practical method for generation expansion planning based on dynamic programming. Electrical Engineering in Japan 112 (8): 114-127.

Sani, A., and D. P. Kroese. 2008. Controlling the number of hiv infectives in a mobile population. Mathematical Biosciences 213 (2): 103-112. TY - JOUR.

Satoh, H., and Y. Serizawa. 1989. Fuzzy decision-making on long-term generation expansion planning. In IFAC Power Systems and Power Control. Seoul,Korea.

Sirikum, J., and A. Techanitisawad. 2006. Power generation expansion planning with emission control: a nonlinear model and a ga-based heuristic approach. International Journal of Energy Research 30 (2): 81-99.

Sirikum, J., A. Techanitisawad, and V. Kachitvichyanukul. 2007, Aug.. A new efficient ga-benders' decomposition method: For power generation expansion planning with emission controls. Power Systems, IEEE Transactions on 22 (3): 1092-1100.

Stremel, J., R. Jenkins, R. Babb, and W. Bayless. 1980, Sept.. Production costing using the cumulant method of representing the equivalent load curve. IEEE Transactions on Power Apparatus and Systems PAS-99 (5): 1947-1956.

Turvey, R., and D. Anderson. 1977. Electricity economics: essays and case studies. United States: Johns Hopkins University Press,Baltimore.

Yildirim, M., K. Erkan, and S. Ozturk. 2006. Power generation expansion planning with adaptive simulated annealing genetic algorithm. International Journal of Energy Research 30 (14): 1188-1199.

\section{AUTHOR BIOGRAPHIES}

RISHABH P. KOTHARI is a graduate student in the Department of Management Science and Engineering at Stanford University. He completed his undergraduate work in the Department of Mechanical Engineering at the Indian Institute of Technology, Bombay. His research interests include modeling and simulation, particularly with applications towards the power and energy sectors.

DIRK P. KROESE has a wide range of publications in simulation and computational statistics, including two monographs: The Cross-Entropy Method (Springer-Verlag, 2004) and Simulation and the Monte Carlo Method, 2nd Edition (Wiley, 2007), jointly with R.Y. Rubinstein. He has held research and teaching positions at Princeton University and the University of Melbourne, and is currently an Australian Professorial Fellow at the School of Mathematics and Physics of the University of Queensland. His email is <kroese@maths.uq.edu.au> 